\title{
Understanding the Formation of the Self-Assembly of Colloidal Copper Nanoparticles by Surfactant: A Molecular Velcro
}

\author{
Raquel Kely Bortoleto-Bugs, ${ }^{1,2,3}$ Talita Mazon,, ${ }^{3,4}$ Márcio Tarozzo Biasoli, ${ }^{1,3}$ \\ Aristides Pavani Filho, ${ }^{1,3}$ Jacobus Willibrordus Swart, ${ }^{3,5}$ and Milton Roque Bugs ${ }^{1,2,3}$ \\ ${ }^{1}$ CTI Renato Archer, Micro Systems Division, Campinas 13069-901, Brazil \\ ${ }^{2}$ Applied Nanotechnology Laboratory, CTI Renato Archer, Campinas 13069-901, Brazil \\ ${ }^{3}$ INCT NAMITEC, Campinas 13069-901, Brazil \\ ${ }^{4}$ CTI Renato Archer, Information Displays Division, Campinas 13069-901, Brazil \\ ${ }^{5}$ Faculty of Electrical and Computer Engineering, CCS/UNICAMP, Campinas 13083-970, Brazil
}

Correspondence should be addressed to Milton Roque Bugs; milton.bugs@cti.gov.br

Received 27 August 2012; Revised 11 January 2013; Accepted 11 January 2013

Academic Editor: Zhenhui Kang

Copyright (C) 2013 Raquel Kely Bortoleto-Bugs et al. This is an open access article distributed under the Creative Commons Attribution License, which permits unrestricted use, distribution, and reproduction in any medium, provided the original work is properly cited.

Self-assembly procedure is employed to synthesize colloidal copper nanoparticles (ccNPs) with cationic surfactant in an environmentally friendly method. Scanning electron microscopy images provide a clear view of the ccNPs formed having an approximate size of $15 \mathrm{~nm}$. The X-ray diffraction reveals that the ccNPs have the two types of copper oxide as well as the metallic copper. The new procedure shows that the cationic surfactant CTAB plays an important role in the understanding and development of self-assembly. There is a strong relationship between the ccNPs formation with the critical micelle concentration of the CTAB which influences both shape and size. The outcomes allowed the development of a molecular model for the ccNPs synthesis showing that the CTAB monomer on the surface has the function of a molecular velcro making the linkage of ccNPs to form an agglomerate with size around $600 \mathrm{~nm}$. Finally, with the emerging new technologies, the synthesis of copper oxide takes a new perspective for their applicability in diverse integrated areas such as the flexible electronics and energy.

\section{Introduction}

Advances in nanotechnology are increasingly dependent on the processes of synthesis to obtain nanoparticles of uniform size and shape [1,2], preferably obtained by environmentally friendly methods [3]. The principle of self-assembly has allowed the appearance of new nanostructured materials with properties that are highly advantageous and suitable to further the development of new processes. Nanoparticles (NPs) with metallic or semiconductive properties have enabled important advances in diverse fields such as in flexible electronics circuits, electronic devices, and alternative energy due to the nanometer scale involved [4-7].

The self-assembly involving metals, reducing agents, and stabilizers such as surfactants hava attracted interest due to the ability of controlling the properties of NPs as uniform size and distribution $[4,8-10]$ through synthesis processes $[11,12]$. However, thermodynamics, kinetics, adsorption, and aggregation make it difficult to control and understand the processes of synthesis using surfactants $[4,13]$. Some effort has been invested in the synthesis of copper I oxide $\left(\mathrm{Cu}_{2} \mathrm{O}\right)$ and copper II oxide $(\mathrm{CuO})$ NPs, which have also attracted interest because of their main characteristic as ptype semiconductors with a range of direct optical bandgap energies and with distinct applications for each oxide [1, $2,8,14,15] \cdot \mathrm{Cu}_{2} \mathrm{O}$ and $\mathrm{CuO}$ have broad applicability as catalysts, batteries, in solar energy conversion, gas sensors, microelectronics, semiconductors, photothermal $[2,16,17]$, and additional applications like antimicrobial activity [18]. Deeper knowledge of the size effects on these systems is of 
great interest because of the control of their optoelectronic properties [17].

The growing interest in environmentally friendly processes is due to pressure on industries in reducing costs and environmental damage with the waste process caused by traditional methods [19]. The procedure using surfactants has developed tremendously because it promotes self-assembly creating possibilities for green synthesis of metallic NPs.

NPs have physicochemical properties that need to be better understood, principally to maintain a standard procedure in obtaining these nanocomposites. However, the size control of nanoparticles is complex and dependent upon parameters such as temperature, ionic strength, $\mathrm{pH}$, and the sequence of reagents addition [10, 20]. Generally, drastic conditions of precipitation are used to collect clusters of NPs in solution which immediately lose their properties. To maintain intrinsic properties of NPs the use of surfactants enables the control of size and shape, increasing the applicability of the NPs $[1,4,7]$.

In this work, we report a new strategy, simple and versatile to synthesize colloidal copper NPs (ccNPs) using a cationic surfactant. The synthesis compared with other processes $[1,21-23]$ has the advantage of being performed in the presence of surfactant in water, under uncontrolled atmospheric conditions, resulting in a homogeneous distribution of the shape and size. The colloidal materials were analyzed with scanning electron microscopy (SEM), absorbance spectroscopy, and X-ray diffraction (XRD). SEM images show substantial difference in the formation of ccNPs in relation to the concentration of surfactant. The crystalline phase of ccNPs displayed by XRD showed the presence of oxides and metallic copper $(\mathrm{Cu})$ which has great technological interest for sintering with intense pulsed light (IPL) [24-27]. In this way, with the results obtained, a molecular model of the synthesis of ccNPs was developed.

\section{Experimental Methods}

2.1. ccNPs Synthesis. The ccNPs were synthesized in aqueous solution, under uncontrolled atmospheric conditions. In a beaker containing ultrapure water, ascorbic acid (Sigma) approximately $420 \mathrm{mM}$ sufficient to adjust the $\mathrm{pH}$ at 2.8 was added. In the solution $1 \mathrm{mM}$ of (1-hexadecyl) trimethylammonium bromide (CTAB-Alfa Aesar) value below critical micelle concentration (CMC) was added. The molar ratio was fixed at 22 times for the above CMC condition. The solution was heated at $55^{\circ} \mathrm{C}$ under constant agitation allowing the equilibrium for $60 \mathrm{~min}$ and then was added slowly to $720 \mathrm{mM}$ of $\mathrm{CuSO}_{4} \cdot 5 \mathrm{H}_{2} \mathrm{O}$ (Alfa Aesar). During the process there is fast and intense color change ranging from yellow, orange, until red-brown indicating the end of the reaction at which point the mixture was left to rest at room temperature for 12 hours. The solution was then centrifuged at 20,000 rpm for 20 minutes at $20^{\circ} \mathrm{C}$, the supernatant was discarded, and the pellet was washed in ethanol, then again centrifuged for five times and dried at room temperature. For the experiment in the absence of CTAB $420 \mathrm{mM}$ of ascorbic acid and $720 \mathrm{mM}$ of $\mathrm{CuSO}_{4} \cdot 5 \mathrm{H}_{2} \mathrm{O}$ was used for the same experimental conditions.
All reagents were of analytical grade and used without further purification.

2.2. Characterization. SEM measurements were carried out with a JEOL SEM-FEG JSM 6330F microscope with a field emission gun and a ZEISS SUPRA 55 microscope with ultrahigh resolution field emission (FESEM). The samples were not sonicated and were prepared by diluting a resultant colloidal solution two-hundredfold with n-isopropanol, placing a drop of the solution onto the silicon wafer and drying it under low vacuum. XRD patterns were collected using a Shimadzu XRD-7000 diffractometer with $\mathrm{Cu} \mathrm{K} \alpha$ radiation at a scanning step of $0.02^{\circ}$ in the $2 \theta$ range from $20^{\circ}$ to $80^{\circ}$. The UV-Vis absorption spectra were measured by Biowave IIWPA spectrophotometer.

\section{Results and Discussion}

The principal result for the ccNPs was obtained in the presence of ascorbic acid (AscAc) with the cationic surfactant $\mathrm{CTAB}$ concentration below the $\mathrm{CMC}$ resulting in the formation of nanostructures containing $\mathrm{CuO}, \mathrm{Cu}_{2} \mathrm{O}$, and $\mathrm{Cu}$ NPs. The SEM images (Figures 1(a), 1(b), and 1(c)) show microspheres obtained in the synthesis process using the solvent water, AscAc, and CTAB with concentration below the CMC. The distribution exhibits homogeneous and nearly spherical structures with a diameter around $600 \mathrm{~nm}$. Figures 1(b) and 1(c) illustrate the top cover of some microspheres that were removed naturally in sample preparation procedure showing the interior completely filled. Figure 1(d) shows the FESEM image of the microspheres and in the enlargement of the highlighted area individual structures can be observed. In short, these structures are the ccNPs formed with an approximate size of $15 \mathrm{~nm}$ and are distributed homogeneously to form the agglomerate of ccNPs (AccNPs) with dimension around $600 \mathrm{~nm}$.

A new experiment was performed to evaluate the contribution of CTAB concentration in the formation of ccNPs. Figure 2 shows SEM images of the ccNPs in a condition above the CMC fixed at 22 times the initial molar ratio. Figures 2(a)2(d) shows a predominant distribution of NPs with irregular surfaces and nonspherical forms as well as random sizes.

To explore the real contribution of AscAc in the ccNPs formation, an experiment under the same conditions was performed in the absence of CTAB surfactant. This condition produced clusters of NPs with an undefined shape as seen in Figure 3(a). In the enlarged SEM Figure 3(b), largest structures formed by clusters of NPs can be observed probably produced by a snowball effect. This effect is responsible for the formation of the clusters that should have neutral charge due to the initial interaction between copper and AscAc.

In order to further characterize the ccNPs, XRD was used to identify the formation of crystalline phases. Figure 4(a) shows the XRD spectrum for the sample of Figure 1 after 3 and 120 days. These XRD measurements were carried out in different time intervals to check the effect of the uncontrolled environment on the stability of the sample. By the analysis of $\mathrm{XRD}$, the spectrum at 3 days shows 


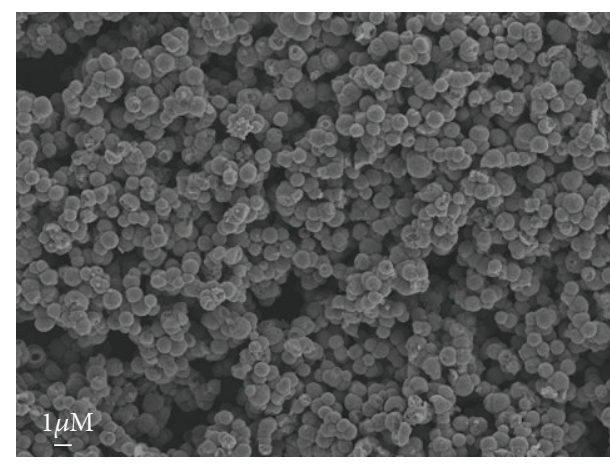

(a)

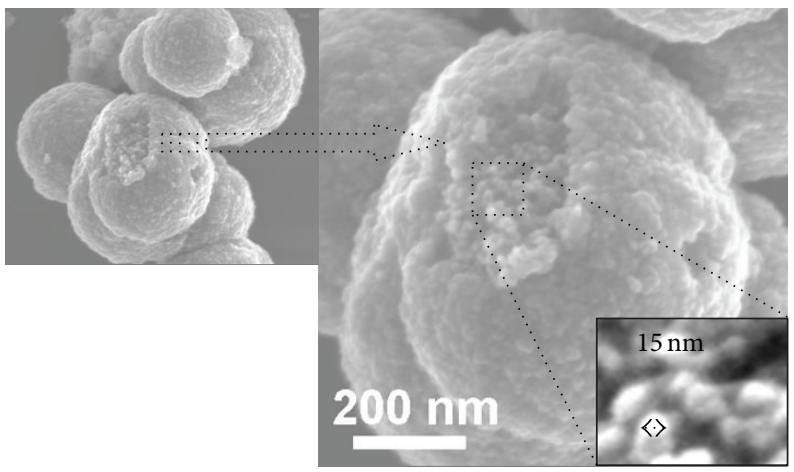

(d)

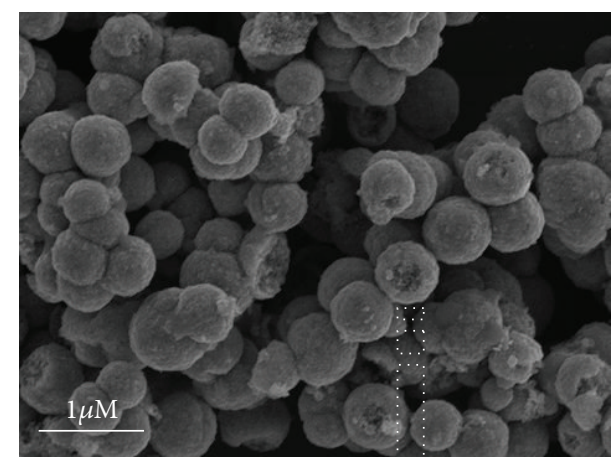

(b)

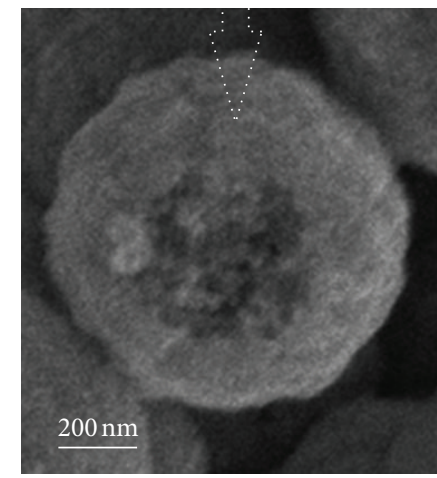

(c)

FIgURE 1: SEM images of the ccNPs with CTAB surfactant concentration below the CMC. (a) The SEM images and further magnified (b and c) show homogeneous and nearly spherical structures with size around $600 \mathrm{~nm}$. Image (c) shows more details in the interior and the surface of the sphere with the top cover removed. (d) FESEM images show details in the enlargement of the highlighted area (inset) with the interior of structures filled with ccNPs showing that copper is incorporated inside the ccNPs with a size around $15 \mathrm{~nm}$.

the presence of $\mathrm{Cu}_{2} \mathrm{O}, \mathrm{CuO}$, and also $\mathrm{Cu}$. The peaks and their respective crystal planes at $2 \theta$ values of $32.5^{\circ}(110)$, $35.5^{\circ}(-111,002), \quad 38.6^{\circ}(111,200), \quad 48.8^{\circ}(-202), \quad 53.4^{\circ}(020)$, $57.8^{\circ}(202), 66.2^{\circ}(-311)$, and $67.9^{\circ}(113)$ indicate the presence of $\mathrm{CuO}$; the peak at $29.6^{\circ}(110), 36.4^{\circ}(111), 42.4^{\circ}(200)$, and $61.4^{\circ}(220)$ corresponds to the $\mathrm{Cu}_{2} \mathrm{O}$ and $\mathrm{Cu}$ peaks appearing in $43.2^{\circ}(111), 50.4^{\circ}(200)$, and $74.1^{\circ}(220)$. All the identified diffraction peaks can be indexed and are consistent with the ICDD data $[24,25,27,28]$. The major differences after 120 days were a significant reduction of the $\mathrm{Cu}_{2} \mathrm{O}$ peaks at $36.4^{\circ}$, $42.4^{\circ}$, and $61.4^{\circ}$, as well as an increase in intensity of the peak at $38.6^{\circ}$ corresponding to $\mathrm{CuO}$ and some peaks of $\mathrm{Cu}$ disappeared with only a weak peak at $43.2^{\circ}$ remaining.

Figure 4(b) displays the absorbance spectrum of ccNPs with CTAB concentrations below and above the CMC. The absorption spectrum below the CMC is formed by a mix of $\mathrm{Cu}$ plus oxide and the sample has a red-brown color. Above the CMC the absorbance seen in the visible shows a strong and characteristic absorption of copper oxidized with a yellowish color $[8,21,23]$.

Self-assembly is a complex process; thereby a comparative analysis of results could contribute to our understanding of the steps involved in the process of synthesis. The formation of the ccNPs begins with the addition of AscAc in water. The initial nucleus is formed in accordance with the supplied concentration and was used in the experiment $420 \mathrm{mM}$ sufficient to maintain $\mathrm{pH}$ at 2.8; however, the AscAc does not have a buffer effect. The growth conditions are a function of Brownian motion, the concentration of AscAc, the agitation, and constant temperature of the medium promoting the formation of symmetric structures which are very similar to the dendrimers [29]. The term dendrimer applies when a molecule is used to form the initial core, but this condition does not occur in our results. The formation of dendrimertype (AscAcDen) starts with AscAc monomers forming a framework maintained by hydrogen bridges. As the concentration of AscAc is relatively higher in molarity, the growth of the dendrimertype occurs according to the availability of free AscAc monomer. Reactive groups on the periphery of AscAcDen are blocked with the addition of the $\mathrm{CTAB}$ monomer determining the size of the ccNPs. The CTAB has a long-chain and small ionic head groups and low aggregation numbers and when alone in solution forms nearly spherical structures below CMC [30]. However, the CMC of CTAB, with values ranging from 0.75 to $1 \mathrm{mM}$ $[31,32]$, depends on the experimental conditions of each environment, such as temperature, ionic strength, and other factors.

In solution, initially, the electrostatic interaction is predominant and an unequal distribution of surfactant mon- 


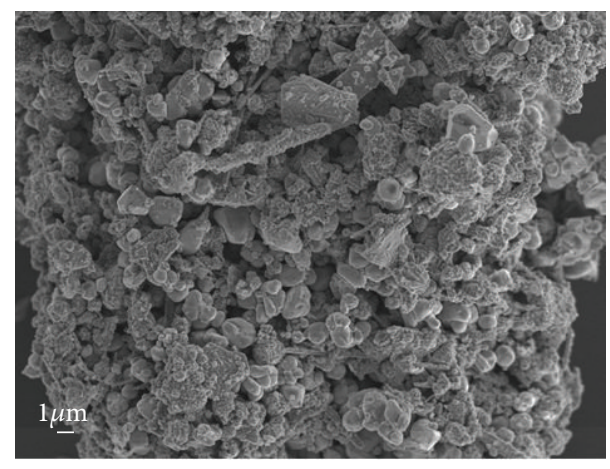

(a)

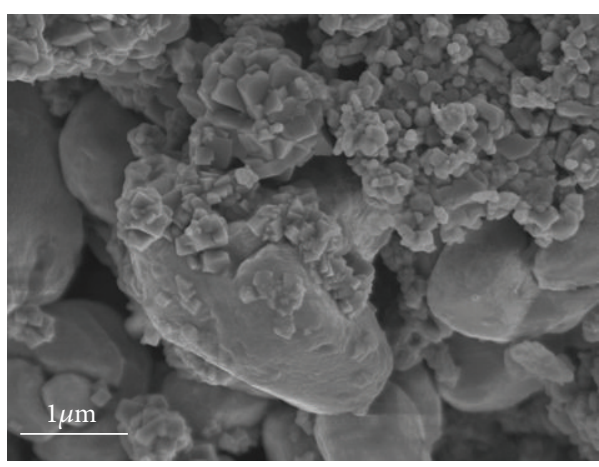

(c)

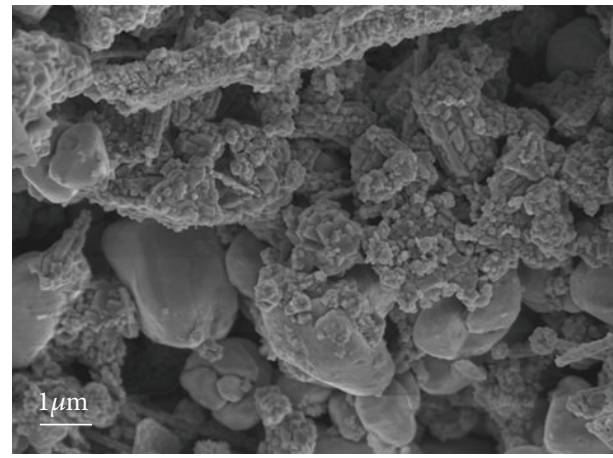

(b)

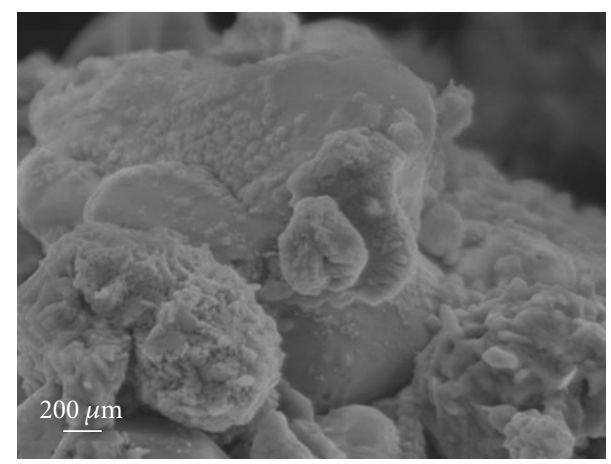

(d)

FIGURE 2: SEM images of the ccNPs with CTAB surfactant concentration above the CMC. All pictures present a distribution of NPs with irregular surfaces and nonspherical forms as well as random size.

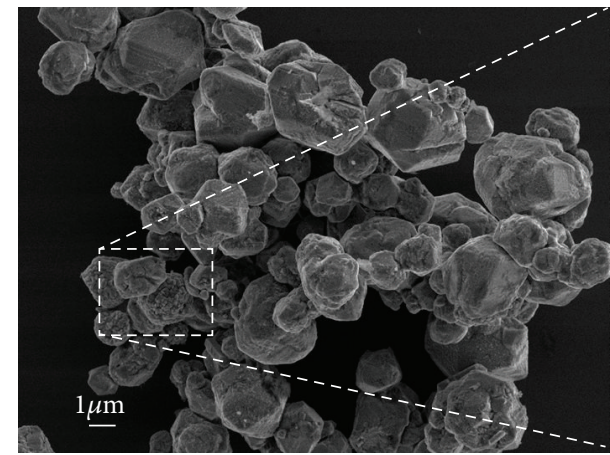

(a)

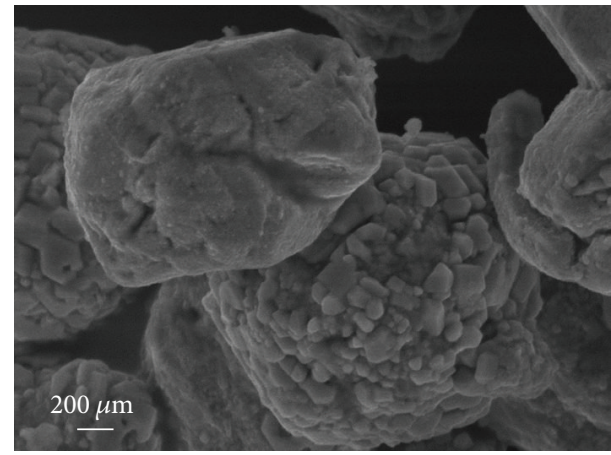

(b)

FiguRE 3: SEM images of the ccNPs without CTAB surfactant. (a) This condition produced structures with irregular shape and further enlarged picture (b) shows structures formed by clusters of NPs.

omers occurs on the periphery of AscAcDen. At this stage, the structures remain formless and the surfactant monomers are attracted electrostatically to the AscAcDen edges, where the polar heads of CTAB bind to the $-\mathrm{OH}$ groups of the AscAc and then the tails accommodate on the dendrimertype in a more hydrophobic region.

The self-assembly of the final shape of ccNPs begins with the addition of copper resulting in an increase of positive charges in the solution. The CTAB alone does not attract copper; however the possibilities of interaction between copper and AscAc are larger and the structures formed are held together only by hydrogen bridges of -OH groups $[5,29]$. The question now is if the copper is bound or if it coordinates a binding because these -OH groups allow the exchange of ligands and create a connection between them that provides a unique environment for the accommodation of the $\mathrm{Cu}$ in 


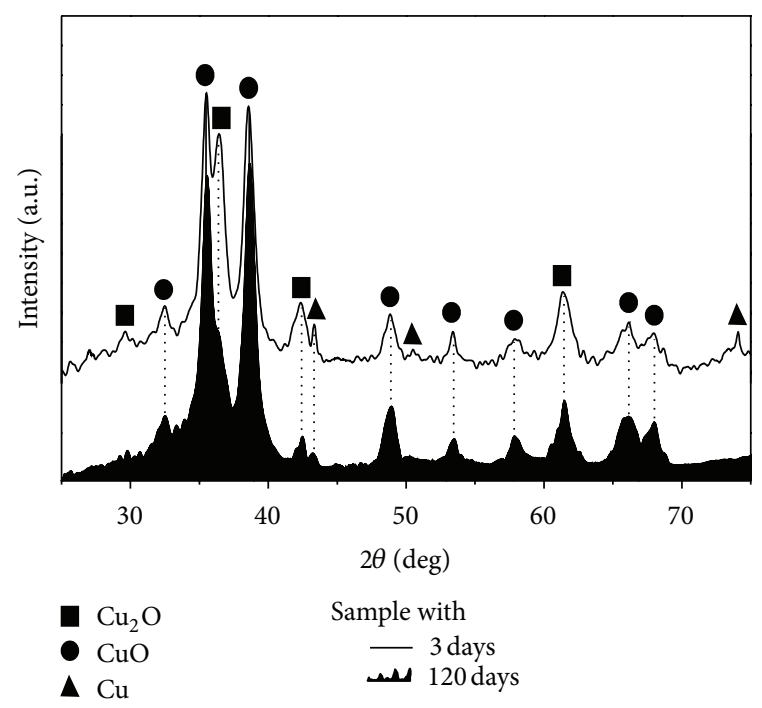

(a)

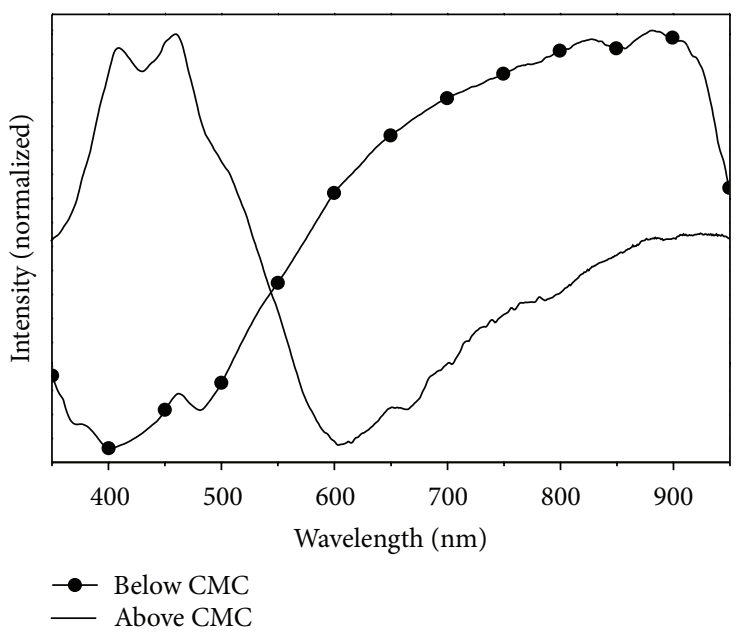

(b)

FIGURE 4: (a) XRD spectrum after 3 and 120 days with CTAB below the CMC shows the diffraction peaks of $\mathrm{Cu}_{2} \mathrm{O}, \mathrm{CuO}$, and $\mathrm{Cu}$. (b) The absorbance spectrum below and above CMC.

the ccNPs. Self-assembly cannot be explained only by the electrostatic interaction and extends far beyond the sum of forces and distribution of charges [10]. In fact, the Brownian motion promotes the linkage of adjacent ccNPs structures and the hydrophobicity of the surfactant tail located on ccNPs favors the bonding of neighboring complexes. This may explain why the size of AccNPs structures is larger than the medium size of dendrimers around 1-100 nm [29]. As shown in Figures 1(c) and 1(d), the interior of the AccNPs is replete of ccNPs with an average size around $15-20 \mathrm{~nm}$, the same size found in the aforementioned result [33].

As the synthesis procedure was done in an acid environment, the $-\mathrm{OH}$ groups of AscAc allow bonds and thus the possibility of oxygen binding to copper is increased, although it has a limited number of internal binding sites in each AscAcDen. Copper may also be located inside the structure and maintained by hydrogen bridges. The cooper oxide surface layer on ccNPs appears to be inevitable because the oxide phases are thermodynamically more stable $[14,21]$. The free cooper binds outside of the ccNPs structure like the oxide and there are probably few regions that allow the interaction of cooper within the structure via electrostatic interaction. For the absorbance spectrum from the solution below CMC, Figure 4(b), the broad envelope has a mix of both copper oxide and $\mathrm{Cu}$ in the ccNPs. The positions of the XRD peaks in Figure 4(a) coincide with the presence of copper oxide closely matched with the absorbance data. The intensity of the peaks corresponding to $\mathrm{Cu}$ is small when compared with the oxide suggesting that only small fraction of $\mathrm{Cu}$ have the possibility of protecting themselves inside the ccNPs structures. Comparing these XRD spectra, there is an evidence of a converted population of $\mathrm{Cu}_{2} \mathrm{O}$ to ionic $\mathrm{CuO}$. The diffusion of oxygen into the oxide is usually a slow process [17]. In addition, flexible electronics is moving forward and requires new approaches that use less energy for
IPL sintering [34-36] and the oxidized NPs with uniform size and shape are much less expensive and stable when compared to $\mathrm{Cu}$ NPs; furthermore the oxidation of the copper NPs is a intrinsic property [14].

Figure 2 shows a variety of shapes including some nanowires and no spherical structures. At higher concentrations, $\mathrm{CTAB}$ becomes rod-like and is spherical at low concentrations [37]; however these data are related only to surfactant systems. When the CTAB is added in the solution, the molecules are immediately driven by sum of electrostatic, hydrophobic, and repulsive forces. The surfactant above the $\mathrm{CMC}$ changes the dynamics of the system and the interaction between CTAB and AscAcDen does not occur as below CMC. In the system, the $-\mathrm{OH}$ groups available are too few to coordinate a binding with copper favoring it to bind with the free oxygen forming copper oxide as shown in the absorbance spectrum in Figure 4(b). Indeed, the excess of surfactant tends to aggregate and these micelles begin to interact with AscAcDen due to the Brownian motion starting the interaction between clusters, so that both are encapsulated or joined to each other. This is evident in Figure 2 where several shapes and sizes can be observed. In the absence of surfactant, Figure 3, only the presence of AscAc and the action of Brownian motion are not sufficient to form structures with spherical shapes.

Previous studies described similar syntheses for obtaining copper NPs with cationic surfactant $[16,38]$, presenting results for synthesis at alkaline $\mathrm{pH}$ with the use of reducing agent such as hydrazine or AscAc. Our experiments were performed at acid $\mathrm{pH}$ and then the surfactant was added in concentration below the CMC resulting in homogeneous NPs.

The CTAB molecules acting as a monomer and their hydrophilic and hydrophobic properties are very important to control the size and shape of the NPs. To complete the 


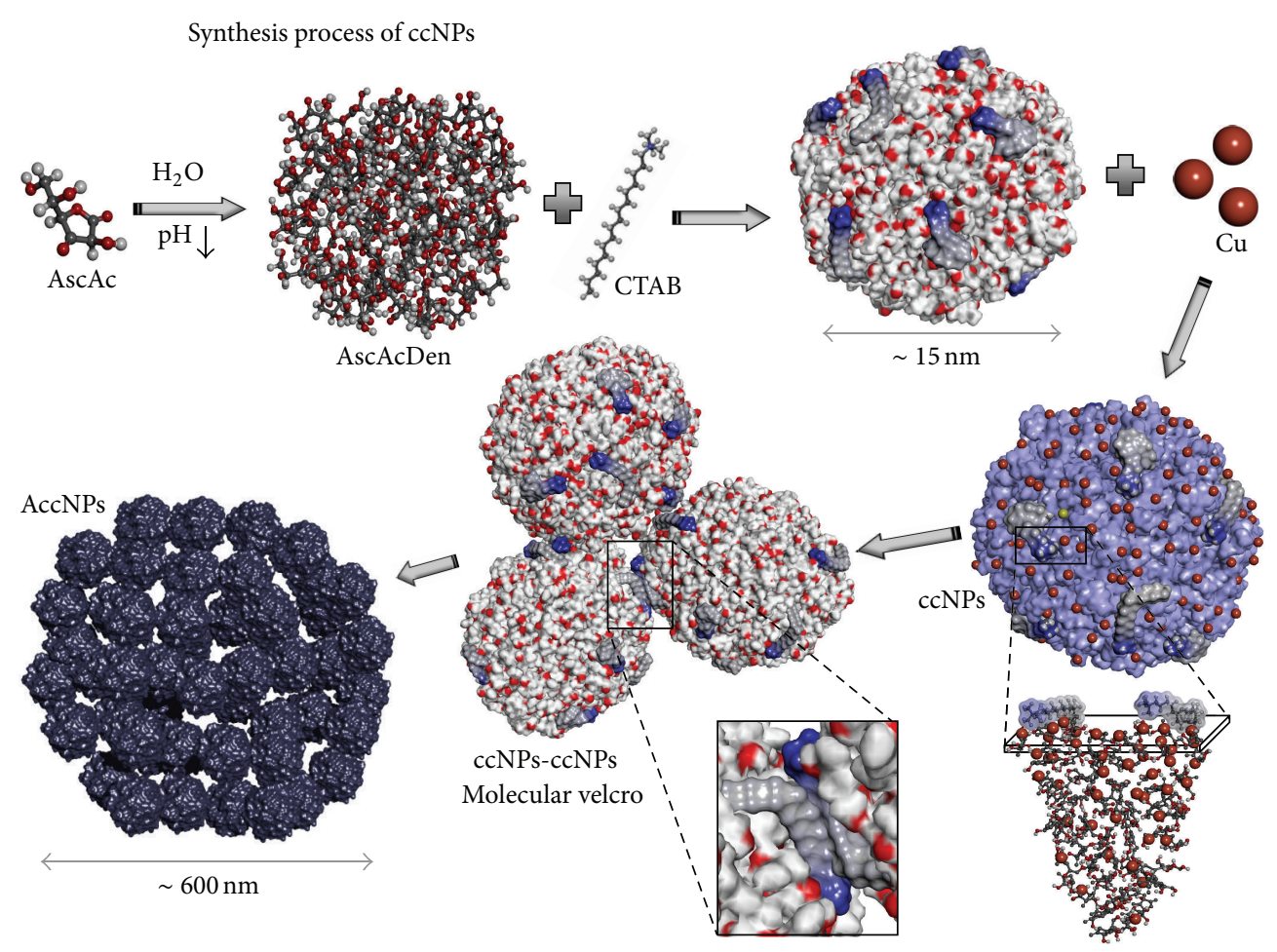

Figure 5: Self-assembly model of the ccNPs. Illustration of the interaction between AscAcDen and CTAB below CMC to form the ccNPs with subsequent addition of $\mathrm{Cu}$. The enlarged pictures show the CTAB located on the AscAcDen and the Cu distributed from the surface into the deeper region. The interactions between the ccNPs were made by a molecular velcro with CTAB tail forming AccNPs. For a better visualization of the structures size relationships were not considered in the molecular ratio.

studies of the synthesis procedures a model was developed for the ccNPs with surfactant concentration below the CMC based on the images of scanning electron microscopy. The proposed model was generated with the DS Visualizer software [39] and is illustrated step by step in Figure 5 according to the reagents addition sequence in the synthesis process. To understand the self-assembly of ccNPs, firstly a certain number of the molecular structure of AscAc were overlapped and then a classical force field was used to optimize the geometry, resulting in a random distribution with the final AscAcDen structure formed only by hydrogen bridges. Afterwards, the addition of the amphiphilic starts the process of electrostatic interaction on the AscAcDen. The CTAB hydrophobic tails efficiently fold on the surface edges in shaft or other hydrophobic regions. At this point, the process of finalizing the ccNPs has not yet occurred, only taking place subsequently with the addition of copper. Looking through a vertical section in ccNPs it can be seen that most of the copper was distributed over the surface and some penetrates closer to the center. The copper on the surface makes it difficult for an upcoming penetration into the interior. The hydrophobic tails distributed over the AscAcDen are exposed to the hydrophilic environment. Energetically, this ambient is unfavorable for the tails needing a hydrophobic environment leading to the approximation of the tails. The joining of the hydrophobic tails forms the molecular velcro resulting in the formation of AccNPs. The linkage phase of the ccNPs by the molecular velcro is named as an intermediate stage in the formation of AccNPs around $600 \mathrm{~nm}$. Considering the size of ccNPs as $15 \mathrm{~nm}$, the volume of $1,767 \mathrm{~nm}^{3}$ can allocate 6,500 AscAc to form the AscAcDen and approximately 64,000 ccNPs to form the AccNPs.

\section{Conclusions}

We developed colloidal copper nanostructures in a new environmentally friendly synthesis. Our results strongly suggest that the self-assembly of the AscAc with CTAB below $\mathrm{CMC}$ form dendrimer-type structures allowing to control the size and homogeneous distribution of the ccNPs. The self-assembly clearly shows that the concentration of cationic surfactant $\mathrm{CTAB}$ plays an important role in controlling the size and shape of the ccNPs. The synthesis procedure resulted in copper oxides NPs that could be used in applications such as sensor sand energy conversion and mainly in the emerging flexible electronics with the possibility of printing directly on the substrate materials with specific properties. The advancement of new technologies such as the IPL sintering technique the formation of oxide stops being a problem and assumes particular attention in the development process. An efficient synthesis can be only considered when producing uniform NPs in size and shape because their physicochemical properties are directly related to these characteristics inasmuch as 
heterogeneous NPs do not have homogeneous properties. To strengthen the results obtained a model of ccNPs formation is proposed showing the action of molecular forces in the selfassembly with the CTAB monomer on the surface making the linkage, a molecular velcro. Our expectations with relation to the process developed are towards the application of the knowledge of self-assembly into new technologies.

\section{Authors' Contribution}

R. K. Bortoleto-Bugs and M. R. Bugs contributed equally to this work.

\section{Acknowledgments}

The work was supported by the CI BRASIL program of the Ministry of Science, Technology and Innovation under Grant no. 301464/2011-1 (R. K. Bortoleto-Bugs) and 301105/2011-1 (M. R. Bugs). The authors gratefully acknowledge the financial support from the INCT NAMITEC $(\mathrm{CNPq}$ no. 573738/2008-4 and FAPESP no. 2008/57862-6), CNPq no. $477367 / 2011-9$, the financial support from CTI Renato Archer/OGU, and the use of SEM facility of the Electron Microscopy Laboratory-LNLS, Campinas-SP, Brazil.

\section{References}

[1] H. Zhang, C. Shen, S. Chen et al., "Morphologies and microstructures of nano-sized $\mathrm{Cu}_{2} \mathrm{O}$ particles using a cetyltrimethylammonium template," Nanotechnology, vol. 16, no. 2, pp. 267272, 2005

[2] D. Gao, J. Zhang, J. Zhu et al., "Vacancy-mediated magnetism in pure copper oxide nanoparticles," Nanoscale Research Letters, vol. 5, no. 4, pp. 769-772, 2010.

[3] M. Poliakoff, J. M. Fitzpatrick, T. R. Farren, and P. T. Anastas, "Green chemistry: science and politics of change," Science, vol. 297, no. 5582, pp. 807-810, 2002.

[4] M. P. Pileni, "The role of soft colloidal templates in controlling the size and shape of inorganic nanocrystals," Nature Materials, vol. 2, no. 3, pp. 145-150, 2003.

[5] R. W. J. Scott, O. M. Wilson, and R. M. Crooks, "Synthesis, characterization, and applications of dendrimer-encapsulated nanoparticles," Journal of Physical Chemistry B, vol. 109, no. 2, pp. 692-704, 2005.

[6] D. Mott, J. Galkowski, L. Wang, J. Luo, and C. J. Zhong, "Synthesis of size-controlled and shaped copper nanoparticles," Langmuir, vol. 23, no. 10, pp. 5740-5745, 2007.

[7] S. Magdassi, M. Grouchko, and A. Kamyshny, "Copper nanoparticles for printed electronics: routes towards achieving oxidation stability," Materials, vol. 3, pp. 4626-4638, 2010.

[8] X. Li, H. Gao, C. J. Murphy, and L. Gou, "Nanoindentation of $\mathrm{Cu}_{2} \mathrm{O}$ nanocubes," Nano Letters, vol. 4, no. 10, pp. 1903-1907, 2004.

[9] J. J. Brege, C. E. Hamilton, C. A. Crouse, and A. R. Barron, "Ultrasmall copper nanoparticles from a hydrophobically immobilized surfactant template," Nano Letters, vol. 9, no. 6, pp. 2239-2242, 2009.

[10] M. Grzelczak, J. Vermant, M. E. Furst, and M. L. Liz-Marzan, "Directed self-assembly of nanoparticles," ACS Nano, vol. 4, pp. 3591-3605, 2010.
[11] K. L. Kelly, E. Coronado, L. L. Zhao, and G. C. Schatz, "The optical properties of metal nanoparticles: the influence of size, shape, and dielectric environment," Journal of Physical Chemistry B, vol. 107, no. 3, pp. 668-677, 2003.

[12] D. Huitink, S. Kundu, C. Park, B. Mallick, J. Z. Huang, and H. Liang, "Nanoparticle shape evolution identified through multivariate statistics," Journal of Physical Chemistry A, vol. 114, no. 17, pp. 5596-5600, 2010.

[13] S. K. Mehta, S. Kumar, S. Chaudhary, K. K. Bhasin, and M. Gradzielski, "Evolution of ZnS nanoparticles via facile $\mathrm{CTAB}$ aqueous micellar solution route: a study on controlling parameters," Nanoscale Research Letters, vol. 4, no. 1, pp. 17-28, 2009.

[14] D. E. Diaz-Droguett, R. Espinoza, and V. M. Fuenzalida, "Copper nanoparticles grown under hydrogen: study of the surface oxide," Applied Surface Science, vol. 257, no. 10, pp. 45974602, 2011.

[15] N. D. Hoa, S. Y. An, N. Q. Dung, N. Van Quy, and D. Kim, "Synthesis of p-type semiconducting cupric oxide thin films and their application to hydrogen detection," Sensors and Actuators $B$, vol. 146, no. 1, pp. 239-244, 2010.

[16] L. Gou and C. J. Murphy, "Solution-phase synthesis of $\mathrm{Cu}_{2} \mathrm{O}$ nanocubes," Nano Letters, vol. 3, no. 2, pp. 231-234, 2003.

[17] M. Yin, C. K. Wu, Y. Lou et al., "Copper oxide nanocrystals," Journal of the American Chemical Society, vol. 127, no. 26, pp. 9506-9511, 2005.

[18] G. Ren, D. Hu, E. W. Cheng, M. A. Vargas-Reus, P. Reip, and R. P. Allaker, "Characterizations of copper oxide nanoparticles for antimicrobial applications," International Journal of Antimicrobial Agents, vol. 33, pp. 587-590, 2009.

[19] C.-J. Li and B. M. Trost, "Green chemistry for chemical synthesis," Proceedings of the National Academy of Sciences of the United States of America, vol. 36, pp. 13197-13202, 2008.

[20] X. Liang, L. Gao, S. Yang, and J. Sun, "Facile synthesis and shape evolution of single-crystal cuprous oxide," Advanced Materials, vol. 21, no. 20, pp. 2068-2071, 2009.

[21] I. Lisiecki, F. Billoudet, and M. P. Pileni, "Control of the shape and the size of copper metallic particles," Journal of Physical Chemistry, vol. 100, no. 10, pp. 4160-4166, 1996.

[22] D. W. Zhang, C. H. Chen, J. Zhang, and F. Ren, "Novel electrochemical milling method to fabricate copper nanoparticles and nanofibers," Chemistry of Materials, vol. 17, no. 21, pp. 52425245, 2005.

[23] M. Blosi, S. Albonetti, M. Dondi, C. Martelli, and G. Baldi, "Microwave-assisted polyol synthesis of $\mathrm{Cu}$ nanoparticles," Journal of Nanoparticle Research, vol. 13, no. 1, pp. 127-138, 2011.

[24] H. S. Kim, S. R. Dhage, D. E. Shim, and H. T. Hahn, "Intense pulsed light sintering of copper nanoink for printed electronics," Applied Physics A, vol. 97, no. 4, pp. 791-798, 2009.

[25] J. Ryu, H. S. Kim, and H. T. Hahn, "Reactive sintering of copper nanoparticles using intense pulsed light for printed electronics," Journal of Electronic Materials, vol. 40, no. 1, pp. 42-50, 2011.

[26] S. Jeong, H. C. Song, W. W. Lee et al., "Stable aqueous based $\mathrm{Cu}$ nanoparticle ink for printing well-defined highly conductive features on a plastic substrate," Langmuir, vol. 27, no. 6, pp. 31443149, 2011.

[27] B. Kang, S. Han, J. Kim, S. Ko, and M. Yang, "One-step fabrication of copper electrode by laser-induced direct local reduction and agglomeration of copper oxide nanoparticles," The Journal of Physical Chemistry C, vol. 115, pp. 23664-23670, 2011. 
[28] H. Wang, Q. Shen, X. Li, and F. Liu, "Fabrication of copper oxide dumbbell-like architectures via the hydrophobic interaction of adsorbed hydrocarbon chains," Langmuir, vol. 25, no. 5, pp. 3152-3158, 2009.

[29] F. Zeng and S. C. Zimmerman, "Dendrimers in supramolecular chemistry: from molecular recognition to self-assembly," Chemical Reviews, vol. 97, no. 5, pp. 1681-1712, 1997.

[30] A. K. Ganguli, A. Ganguly, and S. Vaidya, "Microemulsionbased synthesis of nanocrystalline materials," Chemical Society Reviews, vol. 39, pp. 474-485, 2010.

[31] A. Cifuentes, J. L. Bernal, and J. C. Diez-Masa, "Determination of critical micelle concentration values using capillary electrophoresis instrumentation," Analytical Chemistry, vol. 69, no. 20, pp. 4271-4274, 1997.

[32] M. F. Ottaviani, P. Andechaga, N. J. Turro, and D. A. Tomalia, "Model for the interactions between anionic dendrimers and cationic surfactants by means of the spin probe method," Journal of Physical Chemistry B, vol. 101, no. 31, pp. 6057-6065, 1997.

[33] J. J. J. M. Donners, B. R. Heywood, E. W. Meijer et al., "Amorphous calcium carbonate stabilised by poly(propylene imine) dendrimers," Chemical Communications, no. 19, pp. 1937-1938, 2000.

[34] S. H. Ko, H. Pan, C. P. Grigoropoulos, C. K. Luscombe, J. M. J. Fréchet, and D. Poulikakos, "All-inkjet-printed flexible electronics fabrication on a polymer substrate by low-temperature high-resolution selective laser sintering of metal nanoparticles," Nanotechnology, vol. 18, no. 34, Article ID 345202, 2007.

[35] J. Perelaer, P. J. Smith, D. Mager et al., "Printed electronics: the challenges involved in printing devices, interconnects, and contacts based on inorganic materials," Journal of Materials Chemistry, vol. 20, no. 39, pp. 8446-8453, 2010.

[36] W.-S. Han, J.-M. Hong, H.-S. Kim, and Y.-W. Song, "Multipulsed white light sintering of printed Cu nanoinks," Nanotechnology, vol. 22, Article ID 395705, 2011.

[37] Z. Lin, J. J. Cai, L. E. Scriven, and H. T. Davis, "Sphericalto-wormlike micelle transition in CTAB solutions," Journal of Physical Chemistry, vol. 98, no. 23, pp. 5984-5993, 1994.

[38] S. H. Wu and D. H. Chen, "Synthesis of high-concentration Cu nanoparticles in aqueous CTAB solutions," Journal of Colloid and Interface Science, vol. 273, no. 1, pp. 165-169, 2004.

[39] Accelrys Software Inc., "Discovery Studio Modeling Environment, Release 3.0," San Diego, Calif, USA, 2011. 

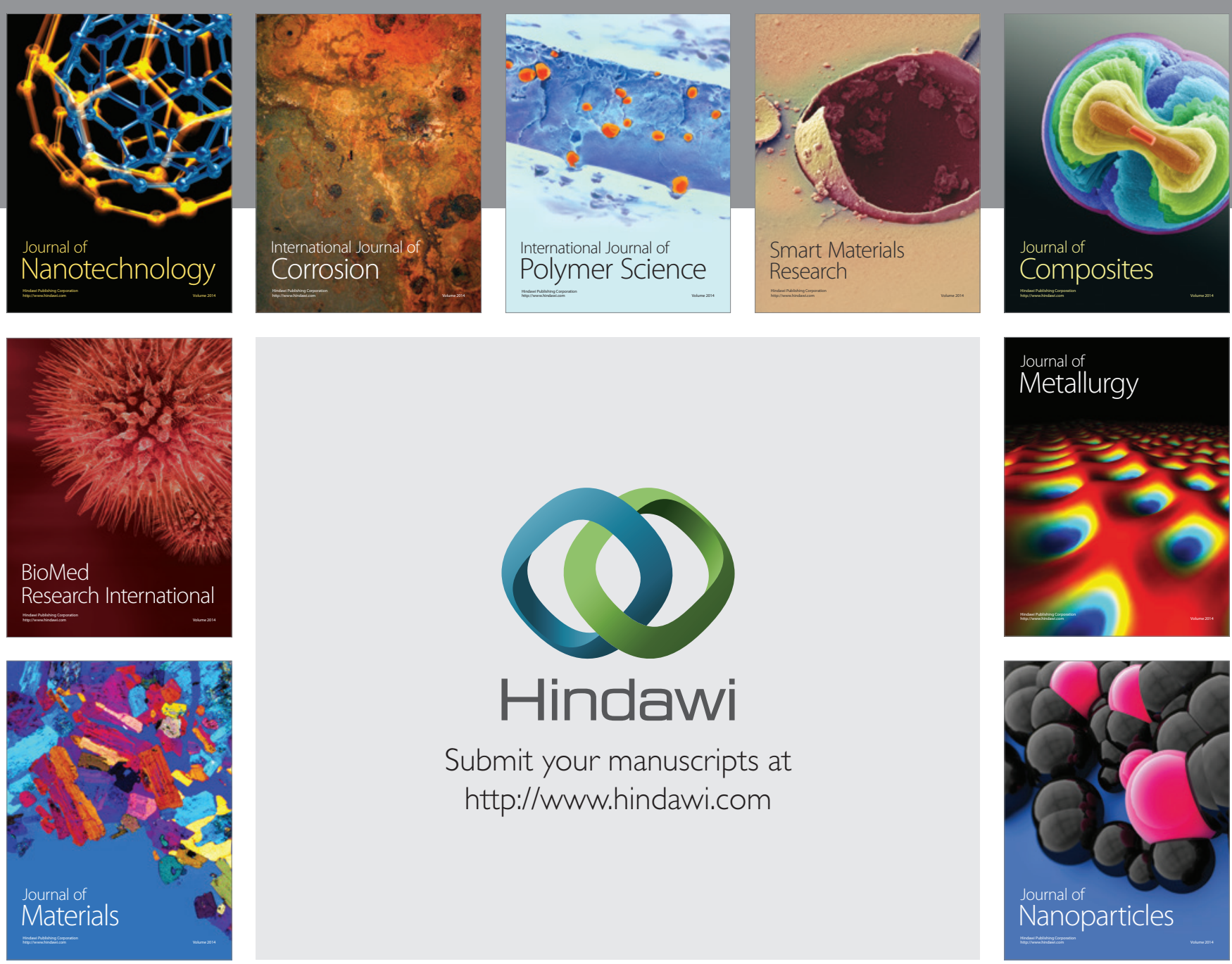

Submit your manuscripts at http://www.hindawi.com
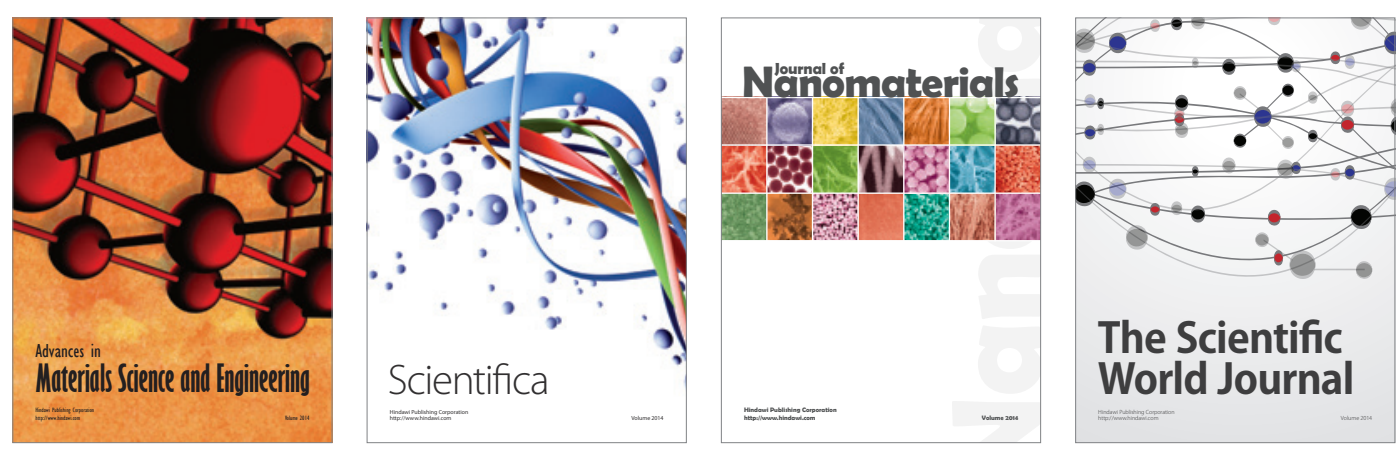

\section{The Scientific World Journal}
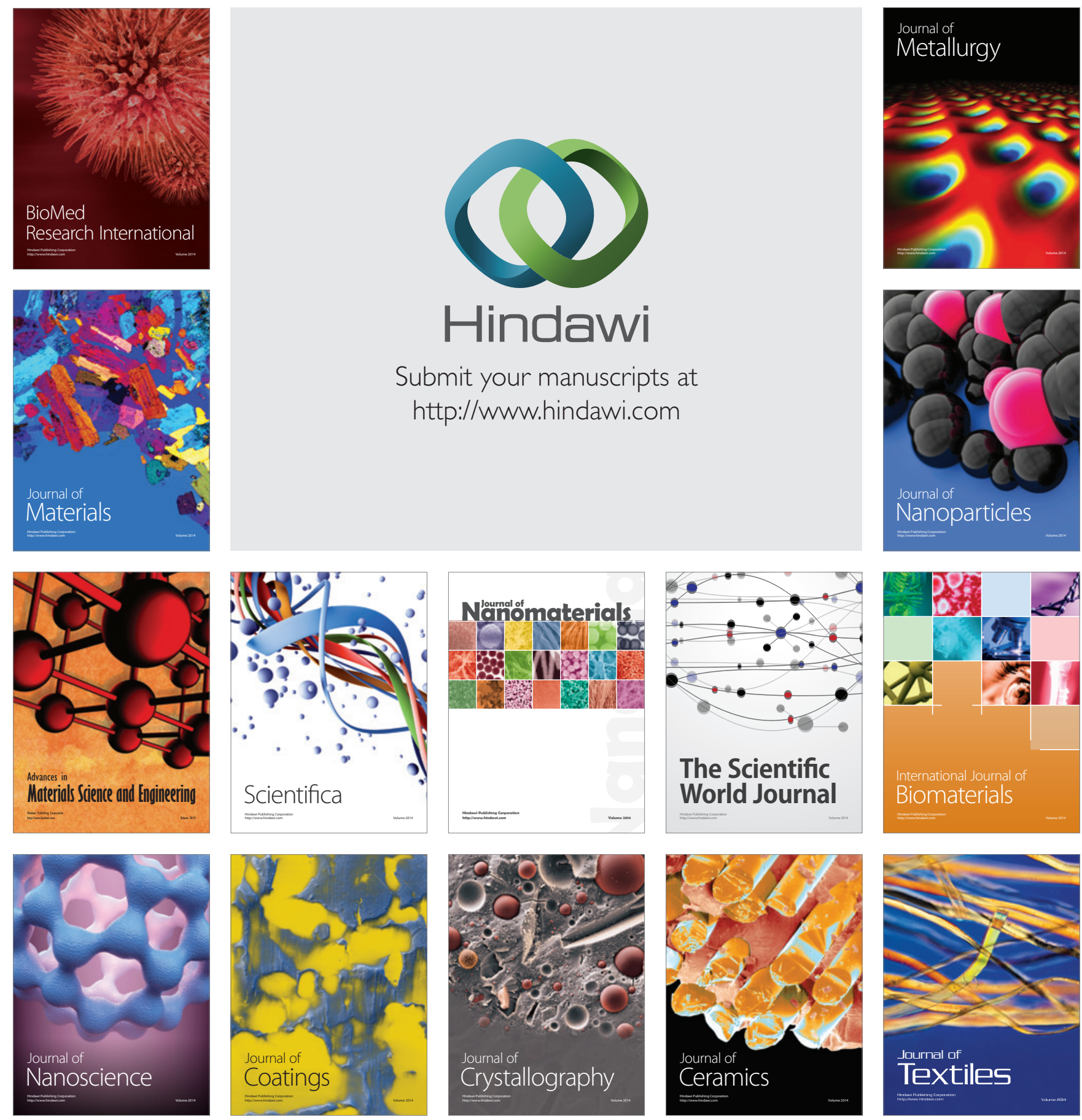